Vol. XXIII No $3 \quad 2017$

\title{
SOFTWARE DEFINE RADIO SYSTEM FOR HF NOISE MEASUREMENTS
}

\author{
Iulian BOULEANU*, Vlad-Cosmin VASILE** \\ * "Nicolae Bălcescu" Land Forces Academy, Sibiu, Romania \\ ** Training Centre for IT and Communication, Sibiu, Romania \\ ibouleanu@gmail.com, vladut.vasile@yahoo.com
}

\begin{abstract}
Accurate noise estimation in the HF band is a defining aspect in the evaluation of SNR for communication links. The reference document that specifies how different types of electromagnetic noise can be taken into account by radio communication system planners is the ITU-R Recommendation P.372.Meanwhile a series of studies have demonstrated that Gaussian noise in the $H F$ band is not always valid because this band noise is strongly affected by atmospheric emission sources such as lightning impulse. This leads to the idea that a distribution suited to express the noise variance is the Bi-Kappa distribution. In addition, over the past decade, SDR devices and real-time spectrum analysers, which can be used to evaluate this noise, have registered explosive growth. This article indicates technical solutions and procedures used to estimate the noise level in the HF subband which can be used for Near Vertical Incidence Skywave (NVIS) communications and proposes a measurement system based on a SDR solution which meets these requirements.
\end{abstract}

Keywords: HF, noise, measurement, methods, SDR

\section{Introduction}

Estimating the noise level in de operation band represents a very important stage in the radio communication planning process. The correct estimation of the noise level allows accurate predictions of the radio link quality based on the signal-noise ratio (SNR) or on BER (Bit Error Rate) which can be computed according to the modulation technique used.

The reference document that specifies how different types of electromagnetic noises can be taken into account by radio communication system planners is the ITUR Recommendation P.372 [1]. Although this document has been updated several times, the reference levels of the noise taken into consideration are based on measurements performed during the 1960s. Today there are two main aspects which impose the revision of the noise level values in the HF band:
- Because of the artificial noise due to the usage of new electric equipment, communication networks, and power networks, it is assumed that the radio noise level has risen compared to the one 50 years ago $[\mathrm{J}]$.

- Some recent studies [2], [3] have reconfirmed that in the high-frequency (HF) band, the assumption of Gaussian noise is not always valid, since noise in this band is strongly affected by impulsive atmospheric radiators, such as lightning. It appears that a modified Bi-Kappa distribution is better suited for modelling such noise.

The noise level can be estimated more accurately today due to the technological development of the last decades, which led to the appearance of more efficient measurement systems, with higher sensitivity and with real-time analysis capabilities [4], [5] but also of procedures 
which are much better adapted in order to capture the effects of the phenomena which contribute to the noise level.

This paper indicates technical solutions and procedures used to estimate the noise level in the HF sub-band which can be used in Near Vertical Incidence Skywave (HF NVIS) communications and proposes a measurement system based on a SDR solution which can meet these requirements.

\section{Particularities of measuring the noise level in the HF NVIS band}

The HF band, whose theoretical limits are situated between 3 and $30 \mathrm{MHz}$, remained in the specialists' attention despite its slow data transfer capacity due to the fact that it allows establishing links at very great distances and due to its attractiveness for governmental communications in emergency situations, when the other types of communication networks can't be used any more [6]. The frequency band which can be used for NVIS communications is even smaller, between 3 and $9 \mathrm{MHz}$ [7], [6], [8] .

The types of radiations contributing to the noise level in the HF NVIS band are: cosmic radiation, lightning, and artificial radiation due to human activity.

The noise level varies according to location and is composed of multiple sources emissions. The noise produced by natural sources is relatively stable during long periods of time and depends less on location. Artificial noise strongly depends on location and time of day, being closely linked to specific human activities in that area (with a radius of up to $10 \mathrm{~km}$ ).

Statistically, in the noise of the HF NVIS band there can be distinguished three types of components, whose properties (Table 1) impose adopting specific measuring techniques.

Because, according to definition [1], the radio noise represents "the combination of a series of electromagnetic signal vectors from unintentional energy sources", the noise analysis of a certain electromagnetic spot has to eliminate all those emissions derived from identifiable singular emission sources.

Due to the possibility of long range propagation, in the HF NVIS band there are many intentional emission sources which produce single carrier noise (SCN). Their emission parameters and the moments these sources start emitting cannot be known a priori because the assignments are made by multiple national authorities and the signal can sometimes be propagated thousands of kilometres. Thus, it is important that the measurement process should take into account measures to eliminate the contribution of such sources [9], [10].

The estimation of impulsive noise (IN) with Bi-Kappa distribution can be done by a rapid sampling of the instantaneous amplitude of the RF signal. The sampling rate has to be according to equation (1):

$f_{s}=\frac{1}{T_{s}} \geq 2 \cdot R B W$

where $f_{s}$ is the sampling frequency, $T_{s}$ is the period between two consecutive samples, and $R B W$ is the filter bandwidth used for measurements.

When establishing the points in which measurements will be performed, the existing noise components have to be taken into consideration. The measuring procedure has to permit the elimination of the contribution of noise of the single carrier type and the identification of impulsive noise. For estimating the WGN contribution, the r.m.s. method can be used [9], [10]. .

The r.m.s. method consists in scanning the frequency band of interest in a series of steps (frequency bins). The values obtained after each sweep will be processed in order to retain only the smallest $20 \%$ of values. The processing results will be recorded in order to allow the statistical analysis of these values during the period of time envisaged by the experiment.

In order to estimate the IN contribution, it is 
necessary to comparatively process the results of two locations whose noises are uncorrelated. This condition assumes that the results should be extracted from the statistical processing of the results obtained by two synchronized systems. The two systems have to be situated far enough, so that the IN component of a location shouldn't be present in the second location, but close enough to assume the same skywave propagation conditions. The method used is called row data sampling and strives characterizing the following IN parameters: peak level, impulse/burst length, impulse/burst period.

Table 1 Properties of radio noise in HF NVIS band

\begin{tabular}{|c|c|c|c|}
\hline $\begin{array}{c}\text { Noise } \\
\text { component }\end{array}$ & Type of sources & Properties & $\begin{array}{c}\text { Distribution and key } \\
\text { parameters }\end{array}$ \\
\hline $\begin{array}{l}\text { Single carrier } \\
\text { noise } \\
(\mathrm{SCN})\end{array}$ & $\begin{array}{l}\text { Radio and wired } \\
\text { communications } \\
\text { sources }\end{array}$ & $\begin{array}{c}\text { Distinct spectral lines } \\
\mathrm{BW}_{\text {noise }}<\mathrm{BW}_{\text {receiver }} \\
\text { Spectral_power } \neq \mathrm{f}(\mathrm{BW})\end{array}$ & \\
\hline $\begin{array}{l}\text { Impulsive } \\
\text { noise } \\
\text { (IN) }\end{array}$ & $\begin{array}{l}\text { Wired electronics, } \\
\text { ignition sparks, gas } \\
\text { lamp starter } \\
\text { lightning }\end{array}$ & $\begin{array}{c}\text { Correlated signals } \\
\mathrm{BW}_{\text {noise }} \geq \mathrm{BW}_{\text {receiver }} \\
\text { Spectral power increases } \\
\text { with }\end{array}$ & $\begin{array}{l}\text { Bi-Kappa distribution } \\
\text { peak level, } \\
\text { impulse/burst length } \\
\text { impulse/burst period }\end{array}$ \\
\hline $\begin{array}{l}\text { White } \\
\text { Gaussian } \\
\text { noise } \\
\text { (WGN) }\end{array}$ & $\begin{array}{l}\text { Wired electronics } \\
\text { and } \\
\text { communications, } \\
\text { cosmic noise }\end{array}$ & $\begin{array}{c}\mathrm{BW}_{\text {noise }} \geq \mathrm{BW}_{\text {receiver }} \\
\text { Uncorellated signals } \\
\text { Spectral power rises with } \\
\mathrm{BW}_{\text {nois }}\end{array}$ & $\begin{array}{l}\text { Normal distribution } \\
\text { r.m.s value of each hour per } \\
\text { day }\end{array}$ \\
\hline
\end{tabular}

The raw data sampling method implies the extraction of at least one sample of each INtype noise specific impulse. Due to the high sampling rate, a great quantity of data will result, which could be an issue for the measuring system. For the statistic estimation of IN it isn't necessary to continually evaluate the frequency/ frequency band of interest. It is recommended [10] to evaluate it for at least $0.5 \mathrm{~s}$, with a sampling rate according to equation (1). After each evaluation, a $30 \mathrm{~s}$ break can be taken, time during which the measuring system can process the measured values.

\section{Measuring systems characteristics}

In order to measure the noise level, there can be used classical spectral analysers or receivers, whit the sweeping method, or modern ones, which perform real-time measurements. The scientific papers of the last years describes less costly and very versatile systems, based on SDR solutions [4], [5]. When it is considered that the noise level is defined as a noise figure (expressed in $\mathrm{dB}$ ) with values exceeding the ones of thermic noise and not as an absolute level, its accurate estimation has to take into account the parameters of all component elements of the receive radio line.

[9] and [10] recommend using two antennas: a reference antenna (short vertical monopole) and an antenna which is specific for the measured domain. The length of the short vertical monopole antenna has to be at least 10 times smaller than the wavelength and need to be placed on a perfectly conductive ground.

When the thermic noise level of the receiver used is less than $10 \mathrm{~dB}$ from the noise level evaluated, a Low Noise Amplifier (LNA) need to be used in order to amplify the signal from the antenna. In this situation, in order to ensure less measurement uncertainties, a $3 \mathrm{~dB}$ attenuator is introduced between the LNA and the receiver/analyser in order to stabilize the line impedance at $50 \Omega$. If the measurement system contains other amplifying or attenuating elements which can be introduced on the signal processing 
route, these will be annulled.

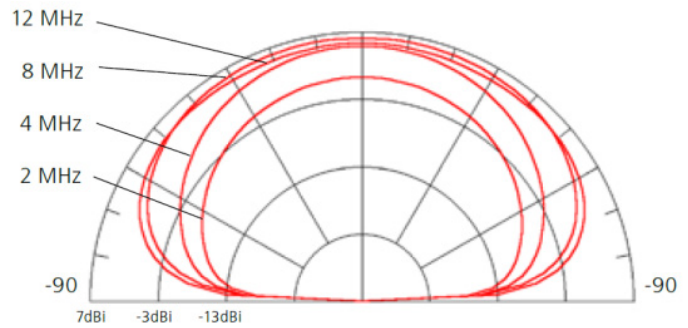

Figure 1 - Radiation pattern in vertical plane for $R F 1938$ antenna

In order to establish the spectral analyser parameters of the measuring system, the following conditions will be taken into account:

- The measuring time depends on the envisaged noise component. For measuring WGN, it is sufficient to scan the frequency (frequency band) of interest once in $10-30$ seconds. When the IN component is included in the noise level, during a scan with a duration of at least $0.5 \mathrm{~s}$ at each $10-30$ $\mathrm{s}$ there have to be taken more samples at a frequency which respects equation (1). In order to take into account the differences between night and day, measurements will be taken for at least 24 hours. To estimate seasonal differences, measurements will be performed on a monthly basis. To estimate the IN contribution, measurements will be performed during working days and during weekends.

- Resolution Bandwidth (RBW) will be $100 \mathrm{~Hz}$ for the case only the RBW component is measured or $100 \mathrm{kHz}$ when the other two components are analysed as well.

- Video Bandwidth (VBW) has to be at least 10 times bigger than $R B W$. It is recommended not to use video filters.

- The detector will be of the r.m.s. type when only the evaluation of WGN is pursued. It has to perform a mediation of the power levels, not of the voltage levels. Applying the raw data sampling method for evaluating the IN component, a peak detector will be used; the processing, including the r.m.s. calculation, will be done later.

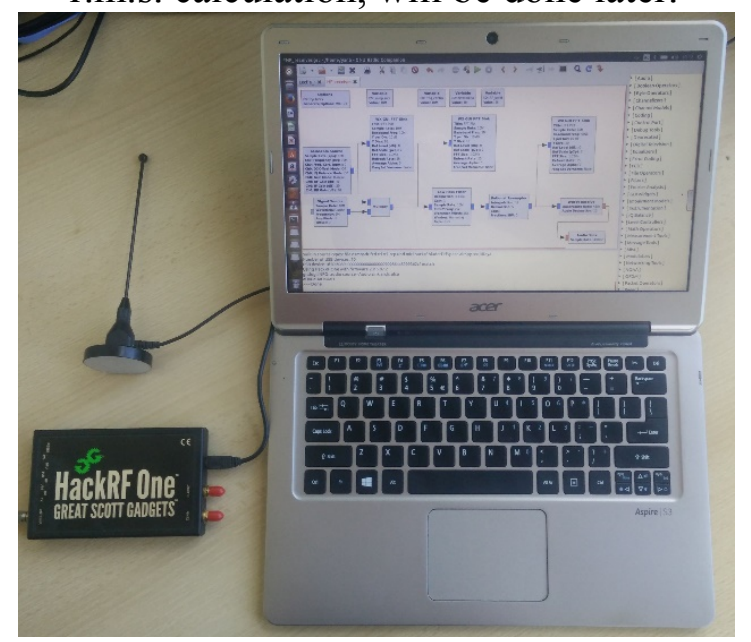

Figure 2 - SDR system with reference antenna

\section{The SDR system for noise level evaluation}

The system developed for noise level evaluation is based on a SDR solution. The system shows a good price-quality ratio. It is based on the specifications of the HackRF One SDR board indicated in Table 2.

Table 2 HackRF One specifications

\begin{tabular}{|c|c|}
\hline Specifications & Values \\
\hline Operating frequency & $1 \ldots 6 \mathrm{GHz}$ \\
\hline Operating mode & Half-duplex \\
\hline Samples per second & up to 20 million \\
\hline Bit resolution & $\begin{array}{l}\text { 8-bit quadrature } \\
\text { samples ( } 8 \text { bit I and } \\
8 \text { bit Q) }\end{array}$ \\
\hline $\begin{array}{l}\text { Application } \\
\text { compatibility }\end{array}$ & $\begin{array}{l}\text { SDR Sharp } \\
\text { GNU Radio } \\
\text { SDR\# }\end{array}$ \\
\hline $\begin{array}{l}\text { Parameters which are } \\
\text { software configurable }\end{array}$ & $\begin{array}{l}\text { RX and TX gain } \\
\text { Baseband filter }\end{array}$ \\
\hline Antenna connector & SMA female \\
\hline $\begin{array}{l}\text { Input and output port } \\
\text { for synchronization }\end{array}$ & Yes \\
\hline Data connection & Hi-speed USB 2.0 \\
\hline USB-powered & Yes \\
\hline Open source hardware & Yes \\
\hline \multicolumn{2}{|c|}{$\begin{array}{l}\text { The directivity characteristic of the RF } \\
1938 \text { antenna is illustrated in Figure } 1 \text { and } \\
\text { the physical system obtained with the shor } \\
\text { vertical monopole antenna is shown in } \\
\text { Figure } 2 \text {. }\end{array}$} \\
\hline
\end{tabular}


Aspire S3 with SSD and I7 processor. For commanding the SDR, the GNU Radio application was used on an Ubuntu 16.04 LTS operating system. The diagram performed with GNU Radio is illustrated in
The second method of signal processing envisages a narrower signal band and thus allows an accurate analysis of the noise level (Figure 5).

Figure 3.

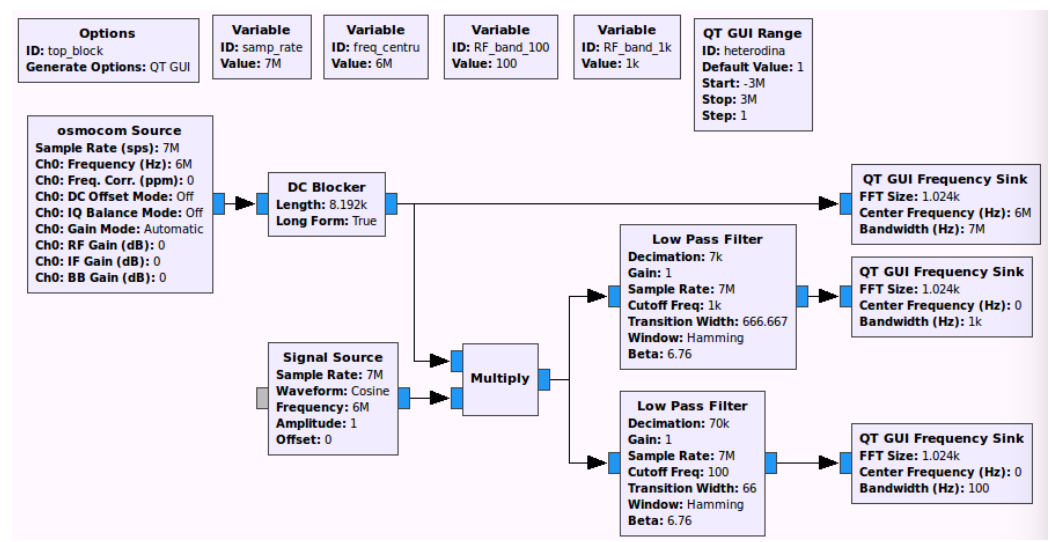

Figure 3: GNU Radio application for HackRF One

This system allows the use of two important signal processing methods. The first one allows the real-time analysis of the entire HF NVIS band ( $3 \mathrm{MHz}-9 \mathrm{MHz}$ ). Because at this analysis a very big RBW is used, the noise level rises very much due to the thermic noise contribution. The results obtained with this method can be processed in order to identify the frequency bands free of signal carriers due to intentional emission sources (Figure 4).

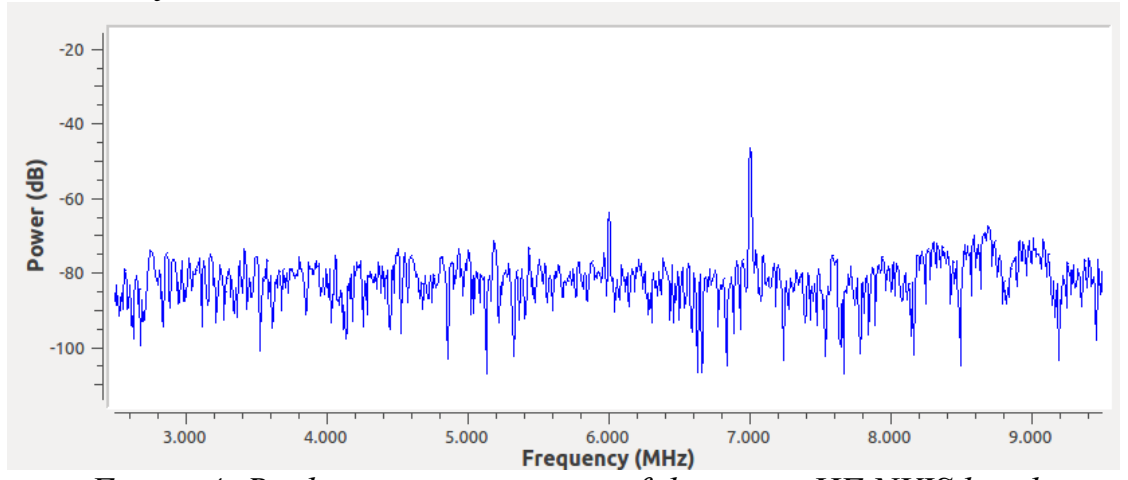

Figure 4: Real-time measurement of the entire HF NVIS band

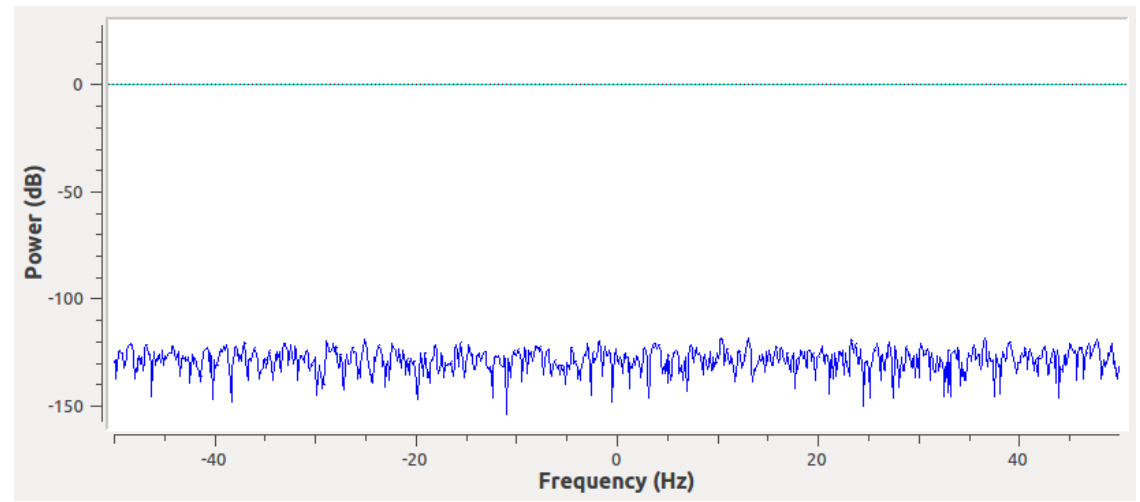

Figure 5: Real-time measurement of a $100 \mathrm{kHz}$ band 


\section{Conclusions}

The paper synthesises methods, procedures and characteristics of systems mentioned in specialty literature for noise level evaluation and transposes them into a SDR based real-time analysis system.

The advantages of such a system consist in its very good price-quality ratio and in the fact that it allows the automation of data measuring and processing. The measuring system can be used in different receive environments and can generate data which can contribute to update the noise level of the HF NVIS band in order to facilitate the planning process of $\mathrm{HF}$ communication lines and of automated modulation recognition systems.

\section{Acknowledgements}

Present research was funded by the Ministry of Education and Research of Romania by UEFISCDI, project code PNII-PT-PCCA-2013-4-0627, contract no. 292/2014 awarded to "Nicolae Balcescu" Land Forces Academy in Sibiu, Romania, for the period 2014-2017.

\section{References}

[1] ITU-R, Recommendation ITU-R P.372-13 - Radio noise, Electronic Publication, https://www.itu.int/rec/R-REC-P.372-13-201609-I/en, Geneva, 2013,

[2] Giesbrecht, J., Clarke R. and Abbott D., "An empirical study of the probability density function of HF noise," Fluctuation and Noise Letters, vol. 6, no. 2, pp. L117-L125, 2006

[3] Giesbrecht J., "Aspects of HF Communications: HF Noise and Signal Features," Ph.D. dissertation, School of Electronic and Electrical Engineering, The University of Adelaide, 2008.

[4] Giesbrecht J., Estimation of SNR for Modulation Recognition in the Presence of HF Noise, 2015 International Conference and Workshop on Computing and Communication (IEMCON), Vancouver, BC, 2015, pp. 1-6. doi: 10.1109/IEMCON.2015.7344433

[5] Bechet P, Miclaus S, Miclaus A, Balint C, Experimental analysis of noise level and channels availability for high frequency OFDM data transmission in NVIS propagation conditions, International Symposium on Electromagnetic Compatibility - EMC EUROPE, 2016, pp. 844-849, 2016

[6] Bouleanu I., Gheorghevici M., Helbet R., „,The quality of the prediction for the NVIS propagation with ITS-HF Propagation", în Electrotehnică, Electronică, Automatică (EEA) nr. 1/2016, ISSN 1582-5175 pp.97-105

[7] Witvliet B. A., et all., Near Vertical Incidence Skywave Propagation: Elevation Angles and Optimum Antenna Height for Horizontal Dipole Antennas, IEEE Antennas and Propagation Magazine, Vol. 57, No. 1, February 2015

[8] Walden M.C., Comparition of propagation predictions and measurements for mildaltitude $\mathrm{HF}$ near-vertical incidence sky wave links at $5 \mathrm{MHz}$, Radio Sci., 47, RS0L09, doi: 10.1029/2011RS004914

[9] ITU-R, Recommendation ITU-R SM.1753-2 - Methods for measurements of radio noise, Electronic Publication, https://www.itu.int/rec/R-REC-SM.1753-2-201209-I/en, Geneva, 2013

[10] ITU-R, Report ITU-R SM.2055 - Radio noise measurements, http://www.itu.int/pub/RREP-SM.2055-2006 